\title{
Isolated asymptomatic elevation of creatine phosphokinase
}

INSERM

\section{Source}

INSERM. (1999). Orphanet: an online rare disease and orphan drug data base. Isolated asymptomatic elevation of creatine phosphokinase. ORPHA:206599

Isolated asymptomatic elevation of creatine phosphokinase is a rare neurologic disease characterized by persistent elevation of the serum creatine phosphokinase (CK) without any clinical, neurophysical or histopathological evidence of neuromuscular disease using the available laboratory procedures. It is usually an incidental finding, diagnosed after exclusion of other possible causes of elevated CK levels. 